\title{
Study on Mobile Meteorological Information Services for Urban Area
}

\author{
Jin-Oh Choi, Member, KIMICS
}

\begin{abstract}
On the limited urban area, precise measurement of meteorological data is not easy for the cost problem. The facilities collecting the data require high installment costs. The mobile sources can be a solution in city region. For example, a public bus on which some meteorological sensors are installed can act as moving information gathering station. The information is gathered on a server and aggregated to generate useful information for smart phone application. To implement the services, several obstacles are exists. This paper studies on a design of this mobile meteorological information service system for urban area.
\end{abstract}

Index Terms - Smart Phone Application, Mobile Sensor, Mobile Meteorological Information Service, USN

\section{INTRODUCTION}

The precise atmospheric and meteorological information in a city area are becoming commonly necessary on our life. A person living in a city area requires the information for several purposes. The information includes temperature, humidity, wind velocity, atmosphere values, and air pollution degree. However, to collect the information, related facilities with the sensors have to be constructed or sensor network system must be installed. These systems require high construction cost and maintenance cost. Therefore a new approach to apply an existing public traffic system as a mobile sensor can be considered.

A private phone can be one approach. If several related sensors are embedded on a phone, it will work as a mobile meteorological center. The information are collected the mobile phones on the road. The mobile phone is possessed by over 3 billion peoples, connected to the internet always, and equipped by many micro sensors. It is becoming true that to accept the mobile phone as a sensor node to build a sensor network.

And the public traffic vehicles can be another attempt [1]. A bus runs routine course periodically. A taxi runs randomly in the urban area. If the vehicles equip some devices, they can act as mobile sensors.

Manuscript received January 12, 2011; revised February 4, 2011; accepted February 7, 2011.

Jin-Oh Choi is with the Department of Embedded IT, Pusan University of Foreign Studies, Pusan, 608-738, Korea (Email: jochoi@pufs.ac.kr)
Figure 1 shows this idea. At a server, the sensing data are collected, processed, and analyzed. The analyzed data can be used to application services reporting air and weather conditions.

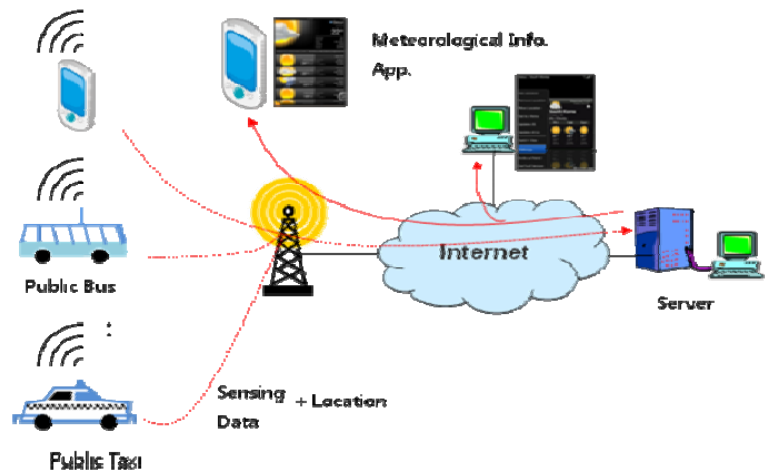

Fig. 1. Mobile Meteorological Information Service Using Mobile Sensors

To make this service possible, first, aggregation method of the gathered sensing data should be introduced. Because the gathered information are LBS(Location Based Services) data, they are generated and cumulated continuously. For successful application services, efficient aggregation techniques are essential. Second, various application user interfaces are developed. Because the gathered information include very precise and real-time data, existing application query types and user interfaces are not sufficient to extract and express the information. Therefore new query types and user interfaces are needed to make use of the aggregated data.

This paper assumes that the sensing data are gathered at a server via USN, 3G N/W or Wi-Fi communication from mobile sensors like as personal phone, public bus, public taxi, and so on. Each mobile sensor equips some atmospheric and meteorological sensors with GPS. The sensing data and location are sent to the server periodically.

The focus of this paper is to design the mobile meteorological information service system for urban area. It includes data gathering methods, data aggregation methods, application query types and user interfaces to analysis and visualize the aggregated data. To generate useful information, all possible query types should be investigated and efficient user interfaces could be developed.

The organization of the remaining parts of the paper is 
follows. Section 2 reviews the related works on the topic and Section 3 introduces the proposing design methods. Finally, the conclusions of this paper are in Section 4.

\section{RELATED WORK}

Generally, the meteorological information service is typical mobile application. It applies the mobile phone to collect the meteorological data especially on large city. An application running on the mobile phone receives meteorological information from a server periodically. Figure 2 show the example, SPB Weather application [2] for some smart phones.

These applications service several simple sensing data on largely covered area, thus it can't provide detail data over small area. This paper focuses on the information service that can provide the precise and various kinds of information on a city area. For the service, data gathering method, data aggregation method, query types, and new user interfaces are developed.

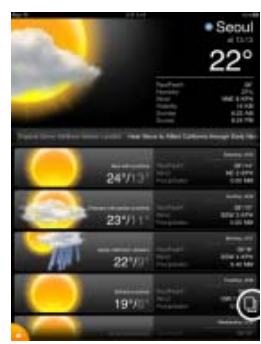

Fig. 2. The Example of Weather Forecasting Service Application on Smart Phone(SPB Weather)

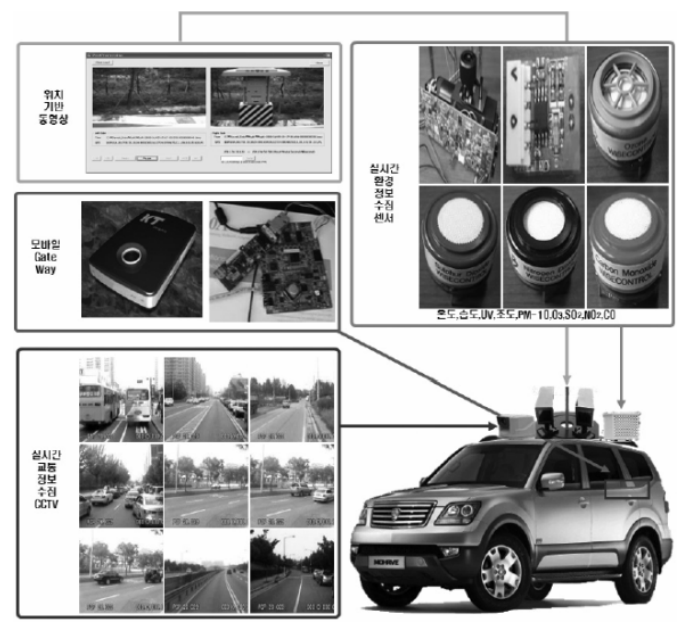

Fig. 3. Mobile USN Atmosphere Measurement Vehicle Equipped with Echo-Sensor(KT)

KT has performed a research [3] which uses an environment measurement vehicle equipped with several echo-sensors. The sensing data can transmitted to a station at real time by mobile USN. Figure 3 shows the vehicle and related equipments. The collected data are analyzed to generate an echo-map. The map is used to investigate atmosphere pollution. This research shows the possibility of the meteorology service using a mobile phone as mobile sensors. But, this approach has a shortage to operate some dedicated sensing vehicles continually and requires high management costs.

This paper shows the system design methods to implement the mobile meteorology information service using mobile sensors bounded on the urban area.

\section{DESIGN OF MOBILE METEOROLOGICAL INFORMATION SERVICE SYSTEM}

Here, the term 'Mobile Meteorological Information Service (MMIS)' is using with two internal meanings. First, the meteorological sensing station is substituted by mobile sensor. Second, the meteorological information service is provided for mobile devices like smart phone.

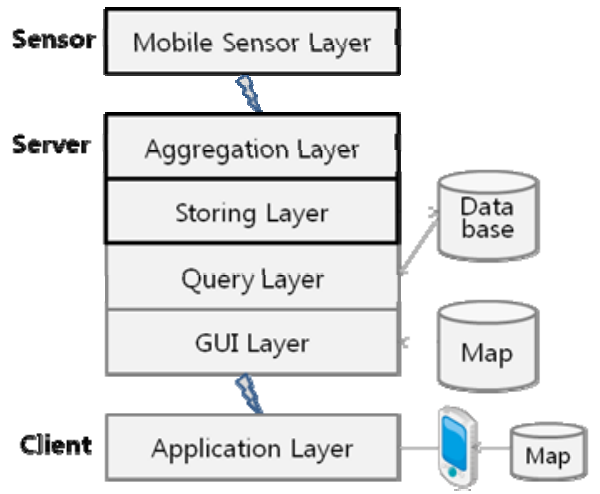

Fig. 4. Layout of MMIS System

Figure 4 shows overall layout and a structure of the MMIS System. The MMIS is consisted of 6 layers. The mobile sensors create meteorological data and transmit them to server via wireless communications, such as USN, $\mathrm{Wi}-\mathrm{Fi}, 3 \mathrm{G} \mathrm{N} / \mathrm{W}$, and so on. The data are stored at database after aggregation step. All possible query types are applied to generate the meteorological information. The derived information, as necessary are merged with map data. Also the mobile application itself can generate much useful information with various data.

The 3 layers emphasized in figure 4, execute their functions continuously in determined time interval. On the contrary, the other 3 layers are called when a request is arrived.

At the mobile sensor layer, the possible sensors are a private phone, public bus, public taxi, and exclusive sensing car. Table 1 shows the benefits and weak points of each sensor. At table 1, private phone seems to be the best, but because of privacy problem, it cannot be acceptable. The next candidate is public taxi. It has no problem with private location exposure and has normal 
management cost. It's better than public bus at city cover ability and accessibility, because public bus runs routine road and stops at night. The public taxi runs random locations in the city, thus covers more wide area. Furthermore, it usually runs during night.

TABLE 1

MOBILE SENSORS (G:Good, N:Normal, B:Bad)

\begin{tabular}{|c|c|c|c|c|c|}
\hline & Privacy & Cost & $\begin{array}{c}\text { City } \\
\text { Cover }\end{array}$ & $\begin{array}{c}\text { Scala } \\
\text { bility }\end{array}$ & $\begin{array}{c}\text { Access } \\
\text { ibility }\end{array}$ \\
\hline Phone & B & N & G & G & G \\
\hline Pub.Bus & G & N & B & G & B \\
\hline Pub.Taxi & G & N & N & G & N \\
\hline Ex.Car & G & B & N & B & G \\
\hline
\end{tabular}

At the aggregation layer, the gathered sensing data at server are aggregated according to certain category. A server has to receive mass sensing data from mobile sensors, and many of them are duplicated. That is, there are many similar sensed data in very near places and on very close times. Furthermore, because the data must be processed on real time, processing efficiency of the data is very important factor. Thus, this layer executes data compaction process.

Because the size of sensing data is increased continuously and rapidly, this step is essential for the efficiency at storing and query layer. The key idea is to calculate an aggregation value of a certain group of collected data. The group is categorized by two factors, time interval (TI) and location zone (LZ). TI is time gap to accumulate, thus if this value is larger, aggregated data size is smaller. LZ is location area to accumulate, thus if this value is larger aggregated data size is smaller.

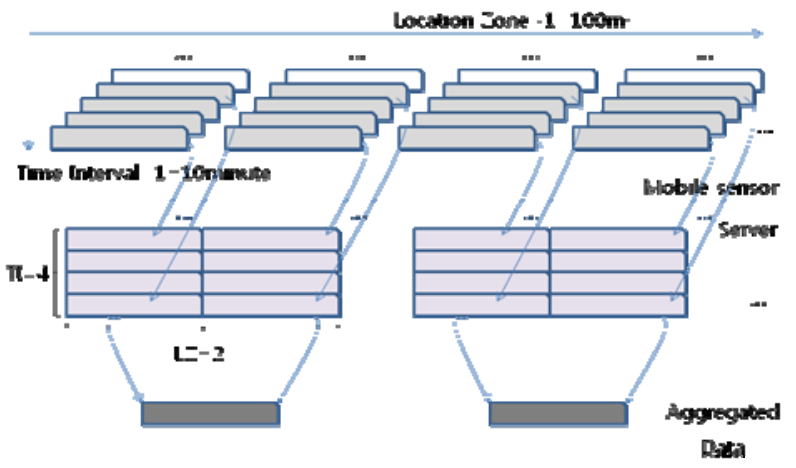

Fig. 5. Example of Aggregation Process

In figure 5, it is assumed that 1 unit of TI is 10 minutes and 1 unit of LZ is 100 square meter. Thus, at the aggregation layer, if TI factor is set as 4 , for a certain LZ, the sensing values are accumulated during 40 minutes then aggregated as 1 value(typically mean value are calculated). And if LZ factor is set as 2, for a certain TI, the sensing values of neighbor pair LZ are accumulated as same group then aggregated as 1 value. The simple calculation of data compaction efficiency is $1 / 8$ as shown in figure 5.

At the storing layer, the aggregated data that calculated at previous layer are stored on a database. The data structure of mobile sensor and this storing data is shown in figure 6.

In figure 6, the data schema of the mobile sensor include sensor ID (for example, taxi ID), exact sensing time, location(for example, GPS coordinator at the sensing time), and sensor values. In figure 6 (b), the storing data schema are aggregated structure of (a), and there are 3 types of data, that is, Agg.Time, Agg.Location, and Agg.Sensor Values. These 3 types will classify the query types later. The Agg.Time is aggregated time interval, and means aggregating period. Then, the Agg.Location is MBR area which the size is decided by $\mathrm{LZ}$ value. The $\mathrm{LZ}$ value are bigger the MBR size is larger. All sensed data are aggregated into corresponding MBR according to its GPS coordinator.

\begin{tabular}{|c|c|c|c|c|c|c|c|c|}
\hline$I D$ & \multirow{2}{*}{\multicolumn{2}{|c|}{$\begin{array}{c}\text { Time } \\
\text { Sensing Time }\end{array}$}} & \multirow{2}{*}{\multicolumn{2}{|c|}{$\begin{array}{l}\text { Location } \\
\text { GPS Coord }\end{array}$}} & \multicolumn{4}{|c|}{ Sensor Value } \\
\hline $\begin{array}{l}\text { Mobile } \\
\text { Sensor ID }\end{array}$ & & & & & Deg. & $\mathrm{Hu}$ & & Dust \\
\hline \multicolumn{9}{|c|}{ 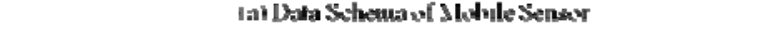 } \\
\hline \multicolumn{2}{|c|}{ Agg Time } & \multicolumn{2}{|c|}{ Agg Location MBR } & \multicolumn{5}{|c|}{ Agg Sensor Value } \\
\hline Start & End & $\begin{array}{l}\text { Left } \\
\text { Top }\end{array}$ & $\begin{array}{l}\text { Right } \\
\text { Bottom }\end{array}$ & Agg Deg & Agg $\mathrm{H}$ & & & Dust \\
\hline
\end{tabular}

Fig. 6. Data Schema Structure

In figure 6, the sensing data generated by mobile sensors that continuously generates sensing signal, and sends sensed data to server periodically. Therefore, the aggregation process and the storing process also are executed periodically. If there are a number of user query requests during the processes, the performance of the server is degraded and severe delay on query will be caused. To overcome this problem there have been many researches related on efficient index. In this paper, discuss on index is out of the focuses.

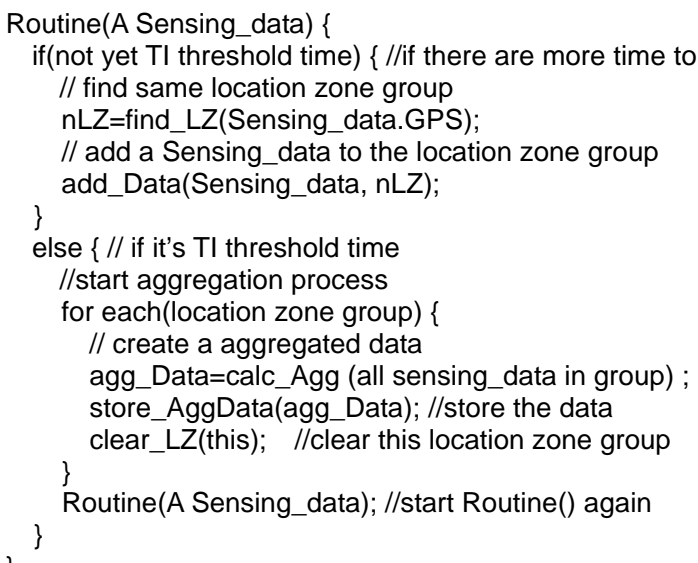

Fig. 7 Aggregation Algorithm

Figure 7 shows aggregation algorithm. In figure 7, 
routine() procedure is called whenever new sensing data are arrived from the mobile sensors. For a sensing data, if time interval threshold is not arrived, the data is added to same location zone group based on its GPS coordinator. Otherwise, aggregation process will start and after reset process, this routine will start again.

At the query layer, useful information is searched from the database storing the aggregated data. Because the aggregated data have 3 kinds of information, the query could be classified as follows: the stored data are composed with a time (set as $\mathrm{X}$ axis), a location (as $\mathrm{Y}$ axis), and sensing values (as $Z$ axis). Then the query types on the storing data would be dependent on two of $X, Y, Z$ axis. That is, all queries will be one of the $\mathrm{X}-\mathrm{Y}-\mathrm{Z}$ pairs. Because 3D GUI is not discussion issue yet, this paper ignores 3 dimensional type queries.

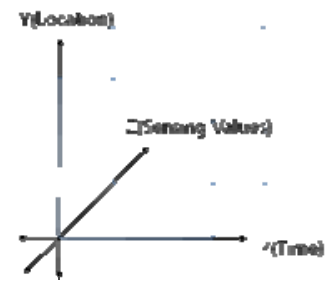

Fig. 8. X-Z Query Example

In figure 8 , most user query type seems to search 2 dimensional planes in a 3 dimensional space. Because the sensing values in $\mathrm{Z}$ axis are multi-values, it may be possible to query some information among the sensing values. This type is 1 dimensional case. All possible query types are shown in table 2.

TABLE 2

QUERY TYPES ON STORING DATA

\begin{tabular}{|c|c|c|c|}
\hline & $\mathrm{X}$ & $\mathrm{Y}$ & $\mathrm{Z}$ \\
\hline $\mathrm{X}$ & $\mathrm{N} / \mathrm{A}$ & Type 4 & Type 1 \\
\hline $\mathrm{Y}$ & Type 4 & N/A & Type 2 \\
\hline $\mathrm{Z}$ & Type 1 & Type 2 & Type 3 \\
\hline
\end{tabular}

In table 2, there are 4 query types. In this application the type 4 is meaningless case because sensing data is absent. Type 3 is 1 dimensional query type. A query about correlation between 2 or more sensing data is an example of this type. Type 1 and Type 2 are 2 dimensional query types. Type 1 gets all or some time and its' sensing values, at certain location. In this figure, the query will be like this: at certain district of a city, during some times ( $\mathrm{X}$ axis), display some sensing values ( $\mathrm{Z}$ axis). The $\mathrm{Z}$ values could be displayed with various user interfaces. The user interfaces could be chart, text table, or mixed form. Figure 9 shows this GUI example of this query type. Here, query results are displayed as chart and table.

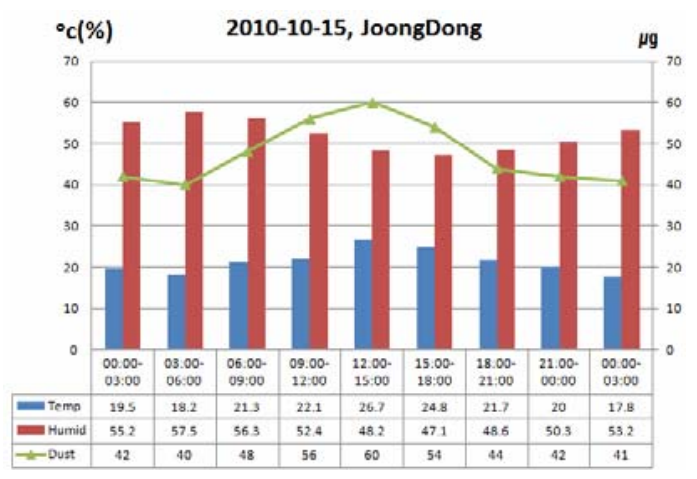

Fig. 9. Type 1 Query Example

Type 2 is Y-Z query, which gets all or some locations and its' sensing values, at certain time. Figure 10 shows this example. In this figure, the query will be like this: at most recent Time Interval, in all districts in a city ( $\mathrm{Y}$ axis), display some sensing values ( $\mathrm{Z}$ axis). The $\mathrm{Z}$ values will be displayed with various GUI (color, text, symbol, etc.).

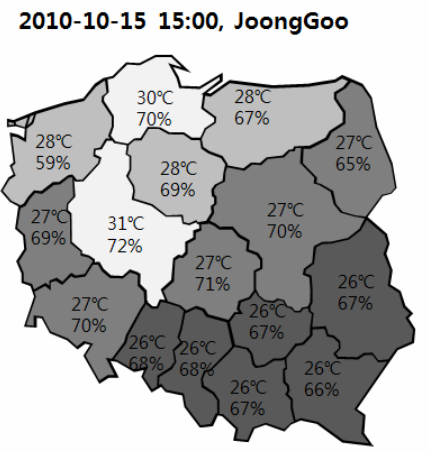

Fig. 10. Type 2 Query Example

At the GUI layer, the queried text information at the previous layer is merged with map to generate visual information. For meteorology map service within city area, to match a location of the sensing data with map is important. The storing data in database have MBR area as location information. If this MBR area is smaller, more precise meteorological information map may be produced.

To create generalized map according to an administrative districts, the MBR location area may be overlaid with administrative district area of a city. In this case, to get a representative value of a district, some interpolation methods should be introduced at the overlapping districts.

\section{CONCLUSIONS}

Over urban area, the needs for atmospheric and meteorological observation values will be increased according to the popularization of smart phone. With the smart phone, a user can scan various atmospheric and meteorological information within the city area. The 
information would be displayed at mobile phone with time and location information. This paper introduced such service system as MMIS (Mobile Meteorological Information Services).

To implement the MMIS, from sensing data gathering problems to GUI generation techniques, many obstacles must be solved. This paper designed the MMIS architecture as 6 layers and proposed several ideas to implement the system. First, various mobile sensor candidates are proposed and evaluated the benefits. And to compact the data size, a very efficient aggregation concept is proposed. The concept is formalized with algorithm. Then, all possible query types on storing sensing data are classified and discussed their characteristics. Though, the environment to use mobile sensor requires many prerequisite conditions, like privacy, cost, cover area, etc., it is clear that, the mobile sensor will be generalized in various application fields, because of its obvious advantages.

Further researches are needed to implement the proposed design and algorithms. The implemented results will be estimated by experiments. The major estimation factors will be the benefit of MMIS system with mobile sensor.

\section{REFERENCES}

[1] P. Ye, S. Kim, I. Lim, J. Choi, J.O. Choi, "The Design of Meteorological Information Gathering System Using Public Traffic System,” 2010 Fall Conference of MICS, Vol. 14, No. 2, pp.110112, 2010

[2] SPB Software : http://www.spbsoftwarehouse.com/

[3] H. Jang, Y. I. Kim, Y. G. Kim, "Role of ICT for Weather Change by Mobile USN Environment Monitoring,” TTA Journal No. 120, 2008.12.

[4] Sensor Web : http://www.opengeospatial.org /projects/groups/ sensorweb

[5] Citysense : http://www.citysense.com/home.php

[6] J. Hu, E. Choi, D. Kim, "Technical Trends of Sensor Network Application,” IITA Weekly Technical Trends, Vol. 1357, 2008.7.

[7] H. Jeong, J. O. Lee, J. Y. Lee, N. Park, G. Jin, B. Kim, "Technical Trends of Sensor Networking," ETRI Electronic Communication Trend Analysis, Vol. 22, No. 3, 2007.6

[8] C. Brenner, M. Sester, "Continuous Generalization for Small Mobile Displays,” Int. Conference on Next Generation Geospatial Information, Boston, MA, pp.19-21, 2003.

[9] K. Leichtenstern, A. Luca, E. Rukzio, "Analysis of built-in mobile phone sensor for supporting interactions with the real World, " in Pervasive Mobile Interaction Devices PERMID 2005.

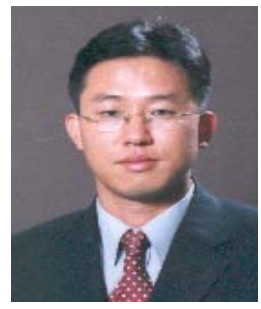

Jin-Oh Choi Received his B.S., M.S., and Ph.D. degree in Department of Computer Engineering from Pusan National University in 1991, 1995, and 2000 respectively. From Jan. 1991 to May 1992, he worked at the Hyundai Electronics as a computer system develop staff. From Mar. 1998 to Feb. 2000, he was a full time lecturor of the Dept. of Information Communication, Kyungdong University. His research areas include mobile GIS, USN application, and meteorological map service. 